\title{
Complications of miliary tuberculosis: low mortality and predictive biomarkers from a UK cohort
}

\author{
Jonathan Underwood ${ }^{1 *}$ (D) Fiona Cresswell ${ }^{2}$, Alex P Salam ${ }^{3}$, Alex J Keeley ${ }^{4}$, Charles Cleland ${ }^{5}$, Laurence John ${ }^{6}$
} and Robert N Davidson ${ }^{6}$

\begin{abstract}
Background: Untreated, miliary tuberculosis (TB) has a mortality approaching $100 \%$. As it is uncommon there is little specific data to guide its management. We report detailed data from a UK cohort of patients with miliary tuberculosis and the associations and predictive ability of admission blood tests with clinical outcomes.

Methods: Routinely collected demographic, clinical, blood, imaging, histopathological and microbiological data were assessed for all patients with miliary TB identified from the London TB register from 2008 to 2012 from Northwest London Hospitals NHS Trust. Multivariable logistic regression was used to assess factors independently associated with the need for critical care intervention. Receiver operator characteristics (ROC) were calculated to assess the discriminatory ability of admission blood tests to predict clinical outcomes.

Results: Fifty-two patients were identified with miliary tuberculosis, of whom $29 \%$ had confirmed central nervous system (CNS) involvement. Magnetic resonance imaging (MRI) was more sensitive than computed tomography (CT) or lumbar puncture for detecting CNS disease. Severe complications were frequent, with 15\% requiring critical care intervention with mechanical ventilation. This was independently associated with admission hyponatraemia and elevated alanine aminotransferase (ALT). Having an admission sodium $\geq 125 \mathrm{mmol} / \mathrm{L}$ and an ALT <180 IU/L had $82 \%$ sensitivity and $100 \%$ specificity for predicting a favourable outcome with an area under the ROC curve (AUC) of 0.91 . Despite the frequency of severe complications, one-year mortality was low at $2 \%$.

Conclusions: Although severe complications of miliary tuberculosis were frequent, mortality was low with timely access to critical care intervention, anti-tuberculous therapy and possibly corticosteroid use. Clinical outcomes could accurately be predicted using routinely collected biochemistry data.
\end{abstract}

Keywords: Tuberculosis, Miliary tuberculosis, Central nervous system infection, Mortality, Biomarkers

\section{Background}

Tuberculosis is one of the leading causes of death from infectious diseases worldwide [1]. Miliary tuberculosis results from widespread haematogenous spread of the Mycobacterium tuberculosis bacilli (TB). Clinical presentations are protean and vary from vague constitutional upset in ambulatory patients to abrupt multi-organ failure [2]. Untreated, miliary TB has a mortality approaching $100 \%$ [3], reducing to $7.1-30 \%$ with treatment $[2,4-6]$.

\footnotetext{
* Correspondence: jonathan.underwood@imperial.ac.uk

${ }^{1}$ Division of Infectious Diseases, Imperial College London, London, UK

Full list of author information is available at the end of the article
}

Miliary TB is uncommon, comprising $1-2 \%$ of TB cases [2], thus specific data regarding its management, particularly the ability to identify patients at high risk of poor outcomes, are lacking [2]. Current treatment guidelines are largely extrapolated from randomised controlled trials of pulmonary $\mathrm{TB}$ rather than specific trials of patients with miliary TB. As such, insights provided by cohort studies are important in guiding best practice. However, most cohorts are small and from the developing world, with limited neuroimaging and microbiological data. We sought to characterise the clinical course, investigation findings, and outcomes of a cohort with miliary TB from an ethnically 
diverse population with one of the highest TB incidences in the UK [7].

\section{Methods}

Participants

Patients with miliary TB were identified from the London TB register (LTBR) from 2008 to 2012 at Northwest London Hospitals NHS Trust. These records were cross-checked against the hospital database of all in-patient admissions.

\section{Data collection}

Diagnosis of miliary TB was confirmed by identification of the characteristic miliary pattern on chest radiograph or computed tomography (CT) along with one or more of the following features: clinical features compatible with $\mathrm{TB}$ and response to anti-tuberculous therapy (ATT); histopathological evidence of TB; microbiological evidence of TB (positive smear or culture). Demographic, clinical, biochemical, radiological, histopathological evidence of TB (caseating or non-caseating granulomas with or without acid-alcohol fast bacilli on Ziehl-Neelson staining), and microbiological data were obtained from the LTBR and the hospital electronic patient records.

\section{Statistical analysis and biomarker selection}

Univariate analysis of continuous data was performed using t-tests and the Mann-Whitney $U$ tests as appropriate. For subsequent biomarker analysis, the primary outcome of interest, defined as an 'unfavourable outcome', was the need for critical care intervention with mechanical intervention. Whereas, a 'favourable outcome' was defined as not requiring critical care intervention with mechanical ventilation.

Multivariable logistic regression $(p<0.1$ for entry from univariable logistic regression) was used to assess factors associated with the need for critical care intervention. Biomarkers that were independently associated with the need for critical care intervention (i.e. an 'unfavourable outcome') in the multivariable model were then assessed for their predictive performance by determining their receiver operator characteristics (ROC). Biomarker classification thresholds were determined by optimising the balance of sensitivity and specificity. Biomarker classification performance was assessed and thresholds determined using the area under the ROC curve (AUC). This is equivalent to the balanced accuracy of the classifier (i.e. accuracy taking account of differences in prevalence of the outcome between groups) for both 'favourable' (i.e. not requiring critical care intervention) and 'unfavourable' outcomes. Additionally, sensitivity, specificity, positive and negative predictive values were calculated. Confidence intervals were determined using bootstrapping with 1000 replicates.

Biomarkers were combined using an exhaustive grid across the range of all possible values and combinations to determine the optimum threshold for each biomarker in combination so that the AUC was maximised.

\section{Biomarker validation}

Given the relatively small size of the dataset, splitting the data into separate training and testing datasets would be subject to bias. Therefore, to validate the predictive rule in this dataset, a leave-one-out-cross-validation (LOOCV) scheme was used. This entailed leaving one subject out of the dataset, then performing an exhaustive grid search, as before, to determine the optimal thresholds for the biomarker(s) of interest. This threshold(s) was then applied to the case that was left out. These steps were then repeated and summary statistics across the range of folds were calculated. Accuracy was determined by the percentage of correct responses across all the cross-validation folds.

\section{Results \\ Baseline characteristics}

Table 1 summarises the demographic data, investigations and clinical outcomes of the cohort of 52 patients with miliary TB. Thirty-six (69\%) had typical miliary changes on chest radiographs with the remainder having miliary appearances confirmed on chest CT. The majority of patients (90\%) were not born in the UK, had no significant comorbidity, and were nearly all (94\%) vitamin D deficient. Half $(14 / 24)$ had tuberculin skin test anergy and $6 \%$ were HIV co-infected. All but one patient (98\%) were admitted to hospital with a median length of stay of 11 days (range 1 - 233 days).

\section{Neuroimaging}

Nineteen patients (37\%) had a CT brain scan performed on their initial admission. Of these, 6 (31\%) had ringenhancing lesions typical of tuberculomas. Eleven patients (21\%) underwent magnetic resonance imaging (MRI) of their brain, of whom 10 (91\%) demonstrated tuberculomas. Notably, 4 patients with normal/nonspecific admission CT had subsequent MRI because due to clinical concern of neurological disease, all of whom demonstrated tuberculomas. The overall prevalence of tuberculomas seen on MRI or CT was 12 of 23 (52\%), but we may have missed lesions in the 29 patients who did not undergo neuroimaging, and in patients in whom tuberculomas developed subsequently.

\section{Microbiology}

Forty patients (77\%) had positive cultures for M. tuberculosis (MTB) complex. Induced sputa lead to a positive culture in thirty (58\%) patients. Only ten sputum samples (19\%) 
Table 1 Baseline characteristics, co-morbidities, admission blood tests, neurological tests and outcomes of a UK cohort of patients with miliary tuberculosis

\begin{tabular}{ll}
\hline Variable & $\begin{array}{l}\mathrm{n}(\%) \text { or } \\
\text { median (range) }\end{array}$ \\
\hline Age (years) & $41(10-87)$
\end{tabular}

\section{Sex}

$$
\text { Male }
$$

\section{Ethnic Origin}

Asian
Black-African
White
Other

Previous diagnosis of TB

Co-morbidities

None

Diabetes

CKD

Anti-TNF therapy

Alcoholism

Heart failure

HIV positive

Hepatitis B sAg positive

Hepatitis C positive

Vitamin D deficient

\section{Admission blood tests}

Sodium (mmol/L)

Creatinine $(\mathrm{mmol} / \mathrm{L})$

Alanine aminotransferase (IU/L)

Alkaline phosphatase (iU/L)

Bilirubin ( $\mu \mathrm{mol} / \mathrm{L})$

Erythrocyte sedimentation rate $(\mathrm{mm} / \mathrm{h})$

C-reactive protein $(\mathrm{mg} / \mathrm{L})$

White cell count $\left(\times 10^{9} / \mathrm{L}\right)$

Neutrophil count $\left(\times 10^{9} / \mathrm{L}\right)$

Platelet count $\left(\times 10^{9} / \mathrm{L}\right)$

Ferritin $(\mu \mathrm{g} / \mathrm{L})$

Vitamin D (nmol/L)

\section{Assessment for CNS involvement}

Number of patients assessed for CNS infection

- CT head consistent with CNS infection

- MRI brain consistent with CNS infection

- Lumbar puncture consistent with CNS infection

Total number with confirmed CNS infection

\section{Outcomes and adverse events}

Death (by one-year)

$32(62 \%)$

$11(21 \%)$

$5(10 \%)$

$4(8 \%)$

$2(4 \%)$

$33(63 \%)$

$6(12 \%)$

$4(8 \%)$

$3(6 \%)$

$3(6 \%)$

$2(4 \%)$

$3 / 49(6 \%)$

$3 / 49(6 \%)$

$1 / 47(2 \%)$

$44 / 57(94 \%)$

132 (106-141)

68 (44-559)

46 (5-453)

$121(39-866)$

$12(4-39)$

$33(1-127)$

$56(3-433)$

$7.1(1.1-17.0)$

$5.3(0.9-15.7)$

238 (53-574)

$522(10-19,070)$

$16(2-117)$

$29(56 \%)$

$6 / 19$ (32\%)

10/11 (91\%)

4/17 (24\%)

$15(29 \%)$

Mechanical ventilation

Table 1 Baseline characteristics, co-morbidities, admission blood tests, neurological tests and outcomes of a UK cohort of patients with miliary tuberculosis (Continued)

\begin{tabular}{ll}
\hline Drug-induced hepatitis & $7(13 \%)$ \\
Seizure & $4(8 \%)$ \\
Pneumothorax & $3(6 \%)$ \\
Haemophagocytic lymphohistiocytosis & $2(4 \%)$ \\
Pericardial effusion & $2(4 \%)$ \\
\hline
\end{tabular}

Data are presented as $\mathrm{n}(\%)$, except for age and blood tests results which are presented as median (range)

Abbreviations: TB Tuberculosis, CKD chronic kidney disease, TNF tumour necrosis factor, HIV human immunodeficiency virus, CNS central nervous system, CT computer tomography; MRI: magnetic resonance imaging, $s A G$ surface antigen

revealed acid-alcohol fast bacilli (AFB) on microscopy. Seventeen patients (33\%) had cerebrospinal fluid (CSF) examination. Of these, four (24\%) had findings compatible with TB meningitis, all of whom subsequently cultured MTB (two of four [50\%] had tuberculomas). Aside from sputa and CSF, MTB was cultured from joint $(n=2)$, pericardial $(n=2)$ and pleural $(n=1)$ fluids $(n=1)$, transbronchial biopsy $(n=1)$ and bone marrow $(n=1)$. Four $(10 \%)$ were resistant to isoniazid, three (6\%) to pyrazinamide, three (6\%) to streptomycin, one to rifampicin and isoniazid (i.e. multidrug resistant - MDR) and none to ethambutol.

\section{Treatment}

All patients were commenced on quadruple ATT with most receiving rifampicin, isoniazid, pyrazinamide and ethambutol. Median (range) duration of ATT was 12 (1.5 - 28) months. Ten (19\%) patients stopped pyrazinamide due to drug-induced liver injury (15\%) or intolerance (4\%). 29 patients (56\%) received corticosteroids during their ATT for a variety of indications including central nervous system (CNS) involvement, systemic inflammatory response syndrome and paradoxical reactions.

\section{Complications}

Follow-up to one-year was complete for 49 patients (94\%). One 87-year-old patient with multiple co-morbidities, including chronic renal failure, died during treatment (after 1.5 months' therapy). One patient died subsequently from constrictive pericarditis secondary to pericardial involvement, two years after successfully completing 12 months of ATT.

Eight patients (15\%) required critical care intervention with mechanical ventilation, seven with respiratory failure and one with neurologic deterioration. Other serious adverse events are shown in Table 1 . Corticosteroid use was more frequent ( $88 \%$ vs. $50 \%, p=0.11$ ) in patients that required mechanical ventilation. In a univariate model, requirement for mechanical ventilation was associated with thrombocytopenia (median platelet count in those 
needing: $148 \times 10^{9} / \mathrm{L}$ vs. not needing mechanical ventilation: $266 \times 10^{9} / \mathrm{L}, p=0.04$ ), hyponatraemia (125 vs. $133 \mathrm{mmol} / \mathrm{L}, p=0.03$ ), hyperbilirubinaemia (17 vs. $11 \mu \mathrm{mol} / \mathrm{L}, p<0.01)$, and increased alanine aminotransferase (ALT: 196 vs. $35 \mathrm{IU} / \mathrm{L}, p<0.001$ ). There were borderline associations with increased erythrocyte sedimentation rate $(37$ vs. $18 \mathrm{~mm} / \mathrm{h}, p=0.08)$ and creactive protein ( $97 \mathrm{vs} 48 \mathrm{mg} / \mathrm{L}, p=0.06$ ) among those requiring mechanical ventilation. There was no significant association between requirement for mechanical ventilation and age $(p=0.72)$, admission vitamin $\mathrm{D}(p=0.39)$, total white cell count $(p=0.14)$, neutrophil count $(p=0.45)$, creatinine $(p=0.34)$, alkaline phosphatase $(p=0.53)$ nor ferritin ( $p=0.54$ but data were only available for 23/52). Additionally, neither comorbidity nor ethnicity were associated with the need for critical care intervention ( $p>0.8$ for all). Whilst there was a trend for a difference in the distribution of ESR between those who did and did not require critical care intervention, in a univariable logistic regression model it was not predictive (odds ratio [95\% confidence intervals]: 0.98 [0.94-1.01] per $100 \mathrm{~mm} / \mathrm{h} ; p=0.19)$. Therefore, ESR was not included in the multivariable model. In a multivariable logistic regression model, only sodium (OR [95\% CIs] 0.19 [0.02-0.93] per $10 \mathrm{mmol} / \mathrm{L}$ reduction, $p=0.04)$ and an ALT $(1.33$ [1.11-1.78] per $10 \mathrm{IU} / \mathrm{L}$ increase, $p<0.0001)$ were independently associated with the need for mechanical ventilation (Table 2).

Admission ALT and sodium were both independently associated with the need for critical care intervention with mechanical ventilation and therefore underwent further ROC analysis to determine predictive thresholds. For the independent prediction of an unfavourable outcome, the optimal threshold for admission ALT was $\geq 180 \mathrm{IU} / \mathrm{L}$ and for admission sodium was $<132 \mathrm{mmol} / \mathrm{L}$ (Fig. 1 and Table 3). The addition of sodium to ALT did not alter classification performance, suggesting that using ALT $\geq 180 \mathrm{IU} / \mathrm{L}$ is sufficient to predict an unfavourable outcome with a positive likelihood ratio of 32.6. Conversely, for the prediction of a favourable outcome, a different threshold for sodium was evident and classification performance was improved by combining ALT <180 IU/L (individual AUC: 0.864 ) and sodium $\geq 125 \mathrm{mmol} / \mathrm{L}$ (individual AUC: 0.670 ) thresholds (combined AUC 0.909, Table 3). It should be noted that this is equivalent to using an ALT threshold $\geq 180$ IU/L or sodium $<125 \mathrm{mmol} / \mathrm{L}$ as criteria from predicting an unfavourable outcome. The relationship between admission sodium and ALT with outcomes is displayed graphically in Fig. 1.

Given the size of the dataset, biomarker prediction validity was assessed using LOOCV. For the prediction of an unfavourable outcome the median (range) ALT threshold was: $\geq 180$ (180-191) IU/L with diagnostic accuracy of $92.3 \%$ across the cross-validation folds. For the prediction of a favourable outcome, the median (range) ALT threshold was <191 (180-200) IU/L; and for sodium was: $\geq 125$ (106-126) mmol/L yielding a diagnostic accuracy of $76.9 \%$ across the cross-validation folds.

\section{Discussion}

We report the lowest one-year mortality from miliary TB (2\%), despite significant rates of severe complications. Additionally, we demonstrate how routinely collected biochemical measures can be used to accurately predict clinical outcomes.

The mortality rate in our cohort compares favourably to previously reported rates of $10-30 \%$ from the developing world and $7.1 \%-21 \%$ from the developed world $[2,4-6]$. Critical care intervention and mechanical ventilation, which was required in $15 \%$ of our cohort, are likely to be responsible for the low mortality we report as these patients would almost certainly have died if these facilities were not available. Additionally, extensive experience in the management of complicated tuberculosis at a high-volume centre are likely to have contributed to our low mortality. Due to the retrospective nature of data collection it was not possible to assess the impact of corticosteroid usage on outcome, and whilst corticosteroid usage was higher in patients requiring mechanical ventilation this may reflect increased rates of CNS involvement and systemic inflammatory response syndrome in patients requiring critical care support. The impact of corticosteroids on mortality in CNS TB infection has been demonstrated, particularly in non HIV-infected individuals [8]. Furthermore, meta-analysis of corticosteroid use in all forms of TB suggest a beneficial effect on mortality, however many of the 41 randomised controlled trials were identified as being

Table 2 Univariable and multivariable logistic regression model results for the outcome of requiring critical care intervention

\begin{tabular}{|c|c|c|c|c|}
\hline \multirow[t]{2}{*}{ Parameter } & \multicolumn{2}{|l|}{ Univariable } & \multicolumn{2}{|l|}{ Multivariable } \\
\hline & Odds ratio $(95 \% \mathrm{Cl})$ & $p$-value & Odds ratio $(95 \% \mathrm{Cl})$ & $p$-value \\
\hline ALT (per $10 \mathrm{IU} / \mathrm{L}$ ) & $1.29(1.13-1.56)$ & $<0.001$ & $1.33(1.11-1.78)$ & $<0.001$ \\
\hline Bilirubin (per $10 \mu \mathrm{mol} / \mathrm{L}$ ) & $2.99(1.29-7.84)$ & 0.01 & $0.69(0.07-3.86)$ & 0.68 \\
\hline CRP (per 10 mg/L) & $1.14(1.02-1.35)$ & 0.02 & $1.13(0.93-1.61)$ & 0.27 \\
\hline Platelet count (per 100 cells/mm3) & $0.39(0.14-0.87)$ & 0.02 & $0.75(0.13-3.60)$ & 0.71 \\
\hline Sodium (per $10 \mathrm{mmol} / \mathrm{L}$ ) & $0.40(0.14-1.03)$ & 0.06 & $0.19(0.02-0.93)$ & 0.04 \\
\hline
\end{tabular}

Abbreviations: ALT alanine aminotransferase, $\mathrm{Cl}$ confidence intervals; CRP c-reactive protein 


\section{a}
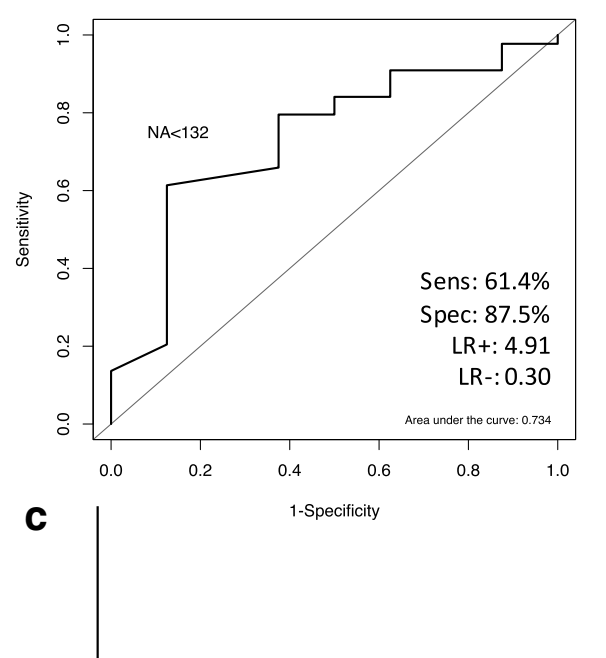

b
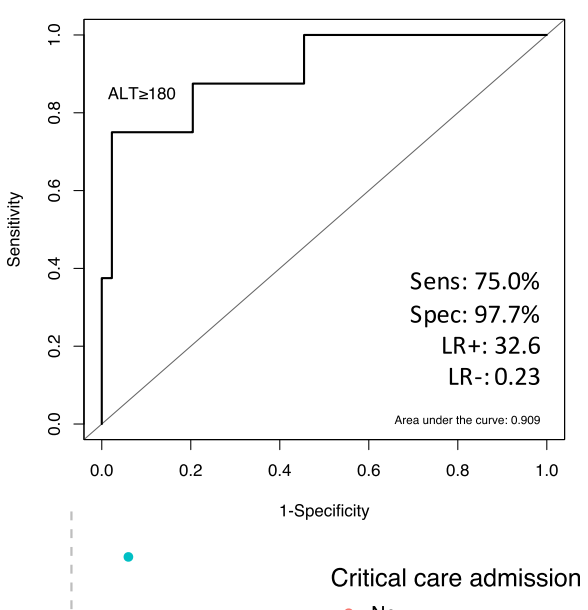

- No

- Yes

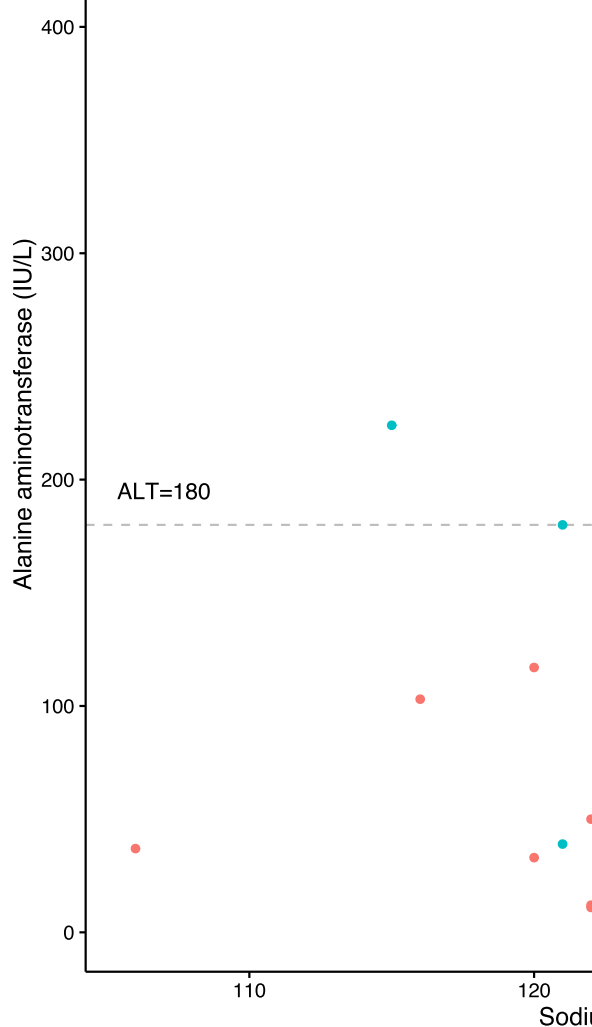

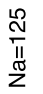

$:$
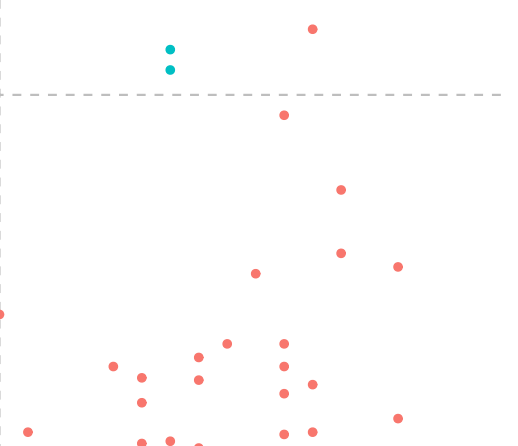

$\bullet$

$\cdot 8$

:

130

Fig. 1 Predictive characteristics of admission sodium and alanine aminotransferase for adverse clinical outcomes. Top: Receiver operator characteristics of admission sodium (a) and alanine aminotransferase (b) for predicting the need for critical care intervention with mechanical intervention with displayed statistics. Bottom: c graphically depicts patients by need for critical care intervention with cut-offs for admission sodium and alanine aminotransferase that identify a favourable outcome with 100\% specificity. Abbreviations: ALT: alanine aminotransferase; Na: sodium; Sens: sensitivity; Spec: specificity; LR+: positive likelihood ratio; LR-: negative likelihood ratio

at high risk of bias, and none were performed to assess corticosteroid usage specifically in miliary TB [9]. Given the multisystem nature of miliary TB with frequent and potentially under-recognised CNS involvement, further research is warranted to clarify the role of corticosteroids.
Hyponatraemia and an elevated ALT on admission were associated with an unfavourable clinical course. The causes of hyponatraemia in those with miliary TB are numerous, but are likely to include brain injury as well as adrenal and pituitary dysfunction. Whereas, an increased ALT identifies those with significant liver injury and in this cohort a 
Table 3 Classification performance of different biomarkers and their optimum threshold by outcome determined using bootstrapping

\begin{tabular}{lllllll}
\hline Predictor & Outcome & Sensitivity (\%) & Specificity (\%) & PPV (\%) & NPV (\%) & AUC \\
\hline ALT $\geq 180$ IU/L & Unfavourable & $75.0(41.7-100)$ & $97.3(92.5-100)$ & $85.7(50.0-100)$ & $95.6(88.6-100)$ & $0.864(0.692-1.00)$ \\
Sodium $<132 \mathrm{mmol} / \mathrm{L}$ & Unfavourable & $87.5(57.1-100)$ & $61.4(46.5-76.1)$ & $29.2(11.1-48.3)$ & $96.4(88.9-100)$ & $0.744(0.583-0.869)$ \\
ALT $<180 \mathrm{IU} / \mathrm{L}$ \& sodium $\geq 125 \mathrm{mmol} / \mathrm{L}$ & Favourable & $81.8(69.8-92.5)$ & $100(100-100)$ & $100(100-100)$ & $50.0(25.0-76.9)$ & $0.909(0.849-0.963)$
\end{tabular}

An unfavourable outcome was defined as the need for critical care intervention with mechanical ventilation, whereas a favourable outcome was defined as not requiring critical care intervention with mechanical ventilation. $95 \%$ confidence intervals determined by bootstrapping (1000 replicates) are in parentheses for each parameter. Abbreviations: ALT alanine aminotransferase, AUC area under the receiver operator curve (equivalent to the balanced accuracy), PPV positive predictive value, NPV negative predictive value

threshold of $\geq 180 \mathrm{IU} / \mathrm{L}$ was strongly predictive of an unfavourable outcome. Both factors are likely surrogates of disease burden, hence their association with clinical outcomes. Our data suggests these routinely collected biochemical measures could be used to identify patients with severe disease at risk of deterioration. However, these thresholds need verifying in an independent dataset. Pending further study, they should be interpreted with caution given the relatively low number of patients in our study. Furthermore, the influence of co-morbidity, in particular HIV-infection and type 2 diabetes given their increasing prevalence in regions with the highest burden of tuberculosis, on the relationship between predictive biomarkers and outcomes needs to be determined.

UK National Institute for Health and Care Excellence (NICE) guidelines [10] currently recommend neuroimaging only for miliary $\mathrm{TB}$ patients with CNS signs or symptoms. Given that miliary TB is caused by the widespread haematogenous dissemination of $M T B$, it is unsurprising that CNS involvement was proven in $29 \%$ of our cohort. However, as not all our patients underwent systematic neuroimaging or CSF sampling, this represents the lower bound of the true prevalence of CNS disease and confirms historical autopsy data documenting high rates of CNS disease [11]. In keeping with other studies, MRI was more sensitive than CT in detecting tuberculomas [4]. CSF examination demonstrated TB meningitis in a minority, suggesting that meningitis may be a late manifestation of disease, however, normal CSF biochemistry and microscopy did not exclude CNS involvement. In addition, CNS tubercles may initially be below the size of detection by CT or MRI, and may paradoxically appear during treatment.

There are no randomised trials to provide guidance on duration of therapy for miliary TB. A 6-month course is currently recommended for miliary TB without CNS involvement in UK, USA, Europe and WHO international guidelines [10, 12-14]. However, as we suspect CNS involvement in miliary TB is often under-recognised, a 6month course of ATT may risk later relapse and complications. Generally, patients in our cohort were treated for 12-months. Whether this contributed to our low mortality is unclear. Given the ubiquity of CNS involvement, we advocate performing contrast-enhanced neuroimaging (ideally MRI) in all patients and CSF examination in those with symptoms or signs of meningitis. Ideally, this should be at the time of diagnosis and possibly repeated 612 weeks later to detect tuberculomas which appear during treatment [15]. In settings where this is not possible, 9-12 months' treatment to cover CNS disease may be prudent. Given the high a priori probability of CNS disease, we advocate consideration for longer empirical duration of therapy (9-12 months) if contrast-enhanced MRI and CSF examination cannot be undertaken, such as in the developing world.

The limitations of this case series include the retrospective and non-systematic nature of initial data collection and incomplete post-treatment follow-up. However, most mortality attributed to miliary $\mathrm{TB}$ occurs in the early stages of disease so it is unlikely that longer followup would significantly change our conclusions $[16,17]$. Additionally, due to the low mortality in our cohort, where there was only one death, we lacked power to detect associations with this important outcome.

\section{Conclusions}

CNS involvement in miliary TB is common and is likely to be under-recognised and under-treated. Although severe complications occur frequently, they can be predicted by admission hyponatraemia and increased ALT. A low oneyear mortality is achievable in a high resource setting with critical care support and mechanical ventilation. Further research is required to establish the role of corticosteroids and prolonged ATT in the management of miliary TB.

\section{Abbreviations \\ ALT: Alanine aminotransferase; ATT: Anti-tuberculous therapy; CNS: Central nervous system; CSF: Cerebrospinal fluid; CT: Computer tomography; HIV: Human immunodeficiency virus; MRI: Magnetic resonance imaging; MTB: Mycobacterium tuberculosis; TB: Tuberculosis}

\section{Acknowledgements}

We gratefully acknowledge the assistance of the TB nurses at Northwest London Hospitals NHS Trust for their assistance with this study.

\section{Funding}

No specific funding for this study was received.

\section{Availability of data and materials}

The raw data are not publicly available due to patients being potentially identifiable but are available (in modified form) from the corresponding author on reasonable request. 


\section{Authors' contributions}

$J U, F C, A P S, L J$ and RND conceived of and designed the study. JU, FC, APS and CC collected data from participating sites. JU conducted statistical analyses. JU, FC and AJK compiled the first draft of the study manuscript and all authors contributed to subsequent revisions. All authors read and approved the final manuscript.

\section{Competing interests}

The authors declare that they have no competing interests.

\section{Consent for publication}

Not applicable.

\section{Ethics approval and consent to participate}

This study was undertaken with the retrospective analysis of routinely collected clinical data which was anonymised before analysis by the researchers. As such, formal Research Ethics Committee review was not required, as confirmed by the NHS Health Research Authority.

\section{Publisher's Note}

Springer Nature remains neutral with regard to jurisdictional claims in published maps and institutional affiliations.

\section{Author details}

'Division of Infectious Diseases, Imperial College London, London, UK. ${ }^{2}$ Lawson Unit, Brighton and Sussex University Hospitals NHS Trust, Brighton, UK. ${ }^{3}$ King's College London, London, UK. ${ }^{4}$ Department of Medicine, Western Sussex Hospitals Trust, Worthing, UK. ${ }^{5}$ Royal Free Hospital, London, UK. ${ }^{6}$ Department of Infectious Diseases, Northwick Park Hospital, Northwest London Teaching Hospitals NHS Trust, London, UK.

Received: 6 August 2016 Accepted: 11 April 2017

Published online: 20 April 2017

\section{References}

1. Murray CJ, Lopez AD. Global mortality, disability, and the contribution of risk factors: global burden of disease study. Lancet. 1997;349:1436-42.

2. Sharma SK, Mohan A, Sharma A, Mitra DK. Miliary tuberculosis: new insights into an old disease. Lancet Infect Dis. 2005;5:415-30.

3. Falk A. U. S. veterans administration-armed forces cooperative study on the chemotherapy of tuberculosis. Tuberculous meningitis in adults, with special reference to survival, neurologic residuals, and work status. Am Rev Respir Dis. 1965:91:823-31.

4. Venkatraman N, King T, Bell D, Woltmann G, Wiselka M, Abubakar I, et al. High levels of neurological involvement but low mortality in miliary tuberculosis: a 6-year case-series from the UK. Eur Respir J. 2016;47:1578-81.

5. Ormerod LP, Horsfield N. Miliary tuberculosis in a high prevalence area of the U.K.: Blackburn 1978-1993. Respir Med. 1995;89:555-7.

6. Touré NO, Cissé MF, Dia Kane Y, Diatta A, Bouker Bakioui B, Ndiaye EHM, et al. Miliary tuberculosis: a report of 49 cases. Rev Mal Respir. 2011;28:312-6.

7. Tuberculosis in the UK, 2014 report. Public Health England (https://www. gov.uk/government/uploads/system/uploads/attachment_data/file/360335/ TB_Annual_report_4_0_300914.pdf); 2014.

8. Prasad K, Singh MB, Ryan $\mathrm{H}$. Corticosteroids for managing tuberculous meningitis. Cochrane Database Syst Rev. 2016:4:CD002244.

9. Critchley JA, Young F, Orton L, Garner P. Corticosteroids for prevention of mortality in people with tuberculosis: a systematic review and meta-analysis. Lancet Infect Dis. 2013:13:223-37.

10. Tuberculosis. National Institute for Health and Care Excellence; 2016 (https://www.nice.org.uk/quidance/ng33/).

11. American College of Physicians. Tuberculoma of the brain. Ann Intern Med. $1934 ; 7: 1141-5$

12. American Thoracic Society - AJRCCM. American Thoracic Society/Centers for Disease Control and Prevention/Infectious Diseases Society of America. Am J Respir Crit Care Med. 2003;167:603-62.

13. Migliori GB, Zellweger JP, Abubakar I, Ibraim E, Caminero JA, De Vries G, et al. European union standards for tuberculosis care. Eur Respir J. 2012:39:807-19.

14. World Health Organization. Guidelines for treatment of tuberculosis. WHO. World Health Organization; 2010. (http://www.who.int/tb/publications/2010/ 9789241547833/en/).
15. Afghani B, Lieberman JM. Paradoxical enlargement or development of intracranial tuberculomas during therapy: case report and review. Clin Infect Dis. 1994;19:1092-9.

16. Lin C-H, Lin C-J, Kuo Y-W, Wang J-Y, Hsu C-L, Chen J-M, et al. Tuberculosis mortality: patient characteristics and causes. BMC Infect Dis BioMed Central. 2014;14:5.

17. Gelb AF, Leffler C, Brewin A, Mascatello V, Lyons HA. Miliary Tuberculosis. American Review of Respiratory Disease. Am Lung Assoc. 1973;108(6):1327-

\section{Submit your next manuscript to BioMed Central} and we will help you at every step:

- We accept pre-submission inquiries

- Our selector tool helps you to find the most relevant journal

- We provide round the clock customer support

- Convenient online submission

- Thorough peer review

- Inclusion in PubMed and all major indexing services

- Maximum visibility for your research

Submit your manuscript at www.biomedcentral.com/submit
Biomed Central 